\title{
DEVELOPMENT OF LANE DEPARTURE WARNING TRAFFIC SYSTEM
}

\author{
P. Balashanmugam ${ }^{1 *}$, G. Balasubramanian ${ }^{2}$, Dipam Talukdar ${ }^{3}$, Rohit roy $^{4}$ \\ ${ }^{*}$ \&2 Assistant Professor, Department of Mechanical Engineering, Annamalai University, Annamalai nagar. \\ ${ }^{3 \& 4}$ UG Students, Department of Mechanical Engineering, Annamalai University, Annamalai nagar. \\ 1 *Email: pbsapme1980@gmail.com
}

*Corresponding Author: -

Email: pbsapme1980@gmail.com

\begin{abstract}
: -
In road-transport terminology, a lane departure warning system (LDWS) is a mechanism designed to warn a driver when the vehicle begins to move out of its lane on freeways and arterial roads. These systems are designed to minimize accidents by addressing the main causes of collisions: driving error, distraction and drowsiness. In this work the authors present a system which has the potential to reduce fatalities which are caused by the common type of crashes called the Run-OffRoad (ROR) crash. The work is to build a lane Departure Warning System for use on roads and highways. The functioning of the system will be to constantly monitor the car placement in relation to the road marks (with the aid of infrared proximity sensor), detect when the driver drifts away from the current lane without an appropriate warn signal, and provide a quick and effective alert to the driver to take a corrective action. To provide an alert system to trigger the quickest response possible. So the aim of this work is to make an intelligent system in hope to design a safer mean of transportation to save lives wasted on roads yearly, especially with the increase in the number of cars introduced to the roads each year. So the need of such security systems in today's intelligent cars is essential.
\end{abstract}

Keywords: - Lane warning departure system, proximity sensor, transportation, infra-red, lane detection

\section{(c) $(\$)$}




\section{INTRODUCTION}

Nowadays, an important social and economic problem is traffic safety. In 1999, about 800,000 people died globally in road related accidents, causing losses of around US \$518 billion [1]. A considerable fraction of these accidents is due to single vehicle lane departure crashes that are mostly caused by inattention, drowsiness, intoxication, incapacitation, unintended steering wheel motions or utilization of cell phones [2]. In this condition, some lane departure warning systems (LDWS) are developed to aid the driver in avoiding or mitigating lane departure crashes through warnings to the driver [2-4]. A warning system should give as much warning time as possible, while triggering few, if any, false alarms.

Currently, the most common approach to preventing lane departure is the use of roadside rumble strips (RRS) on road shoulders. When a vehicle drifts off the road, its tire hits the RRS, which vibrates the vehicle, and makes a loud noise, alerting the driver to take corrective action. However, RRS does require an infrastructure and does not exist in a majority of roadways, and adding RRS can be expensive. Furthermore, RRS pre-to the road boundary guarantees that no lane departures will be missed, yet it increases the frequency of false alarms. Placement further from the road edge reduces false alarms, but decreases the effectiveness of the warning in terms of driver response.

An alternative approach is TLC based method, and when the TLC is below a specified threshold an alarm is triggered. TLC, first proposed by [5], is defined as the time predicted before a specified location on the vehicle will cross the lane boundary. In general, TLC based method provides more warning time than RRS, because warnings are triggered when a driver is predicted to be in danger. However, this prediction can be Wrong, and therefore, the false alarm rates are generally much higher than with RRS. So, RRS has a low false alarm rate, but a short warning time. Conversely, TLC based method, as it is normally implemented, has a much higher false alarm rate than RRS, with a longer warning time. The first step to develop an on-board LDWS is a robust detection of lane boundaries. Numerous methods have been developed to estimate the lane shape and the vehicle position in the lane. They can be classified into region-based methods and edge-based methods.

For a thorough review of lane detection methods, we refer the readers to reference [6].Driver warning systems are designed to help reduce the exposure of occupants to crashes by helping the driver Preemptively avoid them. Lane departure warning (LDW) is a driver warning system designed to help the driver reduce the number of unintended lane departures. Many single vehicle roadway departure crashes take place in light traffic situations and good weather conditions. Such crashes are often due to driver inattention or drowsiness. Drowsiness is frequently reported during night-time driving and in monotonous driving conditions (Horne \& Reyner, 1995), with 51\% of drivers reporting that they have driven a vehicle while feeling drowsy in the past year. NHTSA data indicate that in recent years there have been about 100,000 crashes annually in which police cited driver drowsiness, resulting in about 1,500fatalitie. The goal of a LDW is to warn drivers if they are unintentionally drifting out of their lanes (Figure 1). The system will not take any automatic action to prevent a possible lane departure. Responsibility for the safe operation of the vehicle remains with the driver.

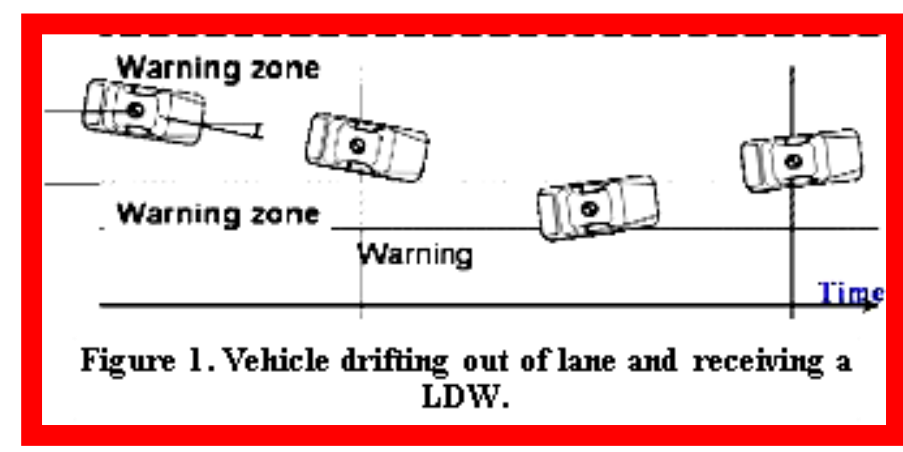

Figure 1: Vehicle is drifting out of the lane and receiving a Lane Departure Warning

\subsection{Types of Lane Departure Warning}

There are two main types of systems:

Systems which warn the driver (lane departure warning, LDW) if the vehicle is leaving its lane (visual, audible, and/or vibration warnings) Systems which warn the driver and, if no action is taken, automatically take steps to ensure the vehicle stays in its lane (lane keeping system, LKS). The first production lane departure warning system in Europe was developed by the United States company Iteris for Mercedes Actros commercial trucks. The system debuted in 2000, and is now available for most trucks sold in Europe. In 2002, the Iteris system became available in Freightliner Trucks' North American vehicles. In both these systems, the driver is warned of unintentional lane departures by an audible rumble strip sound generated on the side of the vehicle drifting out of the lane. No warnings are generated if, before crossing the lane, an active turn signal is given by the driver.

Lane Departure Warning (LDW) System monitors the position of the vehicle with respect to the lane boundary. They warn drivers when the vehicle is travelling above a certain speed threshold and the vehicle's turn signal is not used to indicate the intention of lane change or departure, but nevertheless the vehicle is about to leave its lane. Lane departure warning 
systems are a means of reducing the number of collisions and hence the number of people killed or injured. Several different technologies have been utilized to create LDW systems. The advantage of a camera system is that potential hazardous lane drift is identified ahead of time and warns the driver prior to lane departure occurring. The only drawback of camera systems is that they can be limited by the weather and visibility of the road marking. However, advances in camera technology and the calculation algorithms have resulted in systems that can compensate for adverse road conditions. The infra-red systems are able to detect white lines as well as colored temporary road markings. The infrared system also has the advantage that it is unaffected by poor visibility conditions and is a lower cost system. However, this system cannot predict the vehicle path and therefore can only detect lane departures as the event is occurring.

The majority of LDW systems currently available alert the driver via any combination of the following:

- Warning tone resembling the sound of a vehicle driving to a physical rumble strip. This can also be oriented to only sound on the side of the vehicle that the lane departure is occurring.

- Visual warning on the instrument panel. This can be integrated into the driver's dashboard or located separately.

- Haptic feedback via vibrating the steering wheel or driver's seat, which can vibrate on the side of the seat that the lane departure is occurring.

It is also important that the driver is informed when the system is not functioning correctly or temporarily unavailable. One complication in the design of the systems is that an effective lane departure warning system will need to function irrespective of the lane boundary type, while the boundary geometry differs from country to country.

\subsection{Purposes}

In this work the Lane Departure Warning System in use as a prototype for tomorrow's intelligent car system. The main purpose of this system is to detect the lanes on the road and thus to avoid accidents, it is something which is associated with the safety for the traveller. The basic design includes infrared sensors consists of infrared emitters and infer red receiver, transmitter receiver depending upon the sensor output, the LCD will display the information to the driver.

\section{Previous work}

One of the earliest works, by Pipes [7], modelled the driver as again and a time delay, and modeled the vehicle lateral position as an integration of steering wheel angle. Over the next few decades, that work expands upon, as the model of the driver became more complicated and attempted to take into account evidence provided by studies of driver behaviour.

Weirwille [8], who has been active in this field for many years, presented an early model which took into account past lateral displacement, future roadway curvature, and driver vantage point. This work showed that information on the upcoming road curvature helps to eliminate the effects of perceptual and reactions lag. The main assumption in control theory approaches to driver modelling is that humans, and the vehicles that they control, can be adequately simulated using 2nd order systems. Stochastic and non-linear effects, such as crosswind response, cannot be modeled well using these approaches. Furthermore, it becomes very difficult to take into account environmental effects such as the presence of other vehicles. One area where these approaches have worked well is in car following, as shown by Bekey [9], Chandler [10], Ioannou [11], and Naab [12].

The Daisy system, developed by Feraric and Onken [13], uses multiple Fuzzy-ART models for different low level situations such as lane changes, passing, cruising, etc., and attempts to learn an individual driver's acceptable levels of Time to Lane Crossing (TLC) and Time to Collision (TTC). A separate model was used to determine what the driver's intent would be in a given situation, to select an appropriate low level model. Heuristic approaches have also been used, where rules are applied to state variables of the type: If the lateral position > threshold, then sound alarm, such as the work by Isomoto [14]. Pilutti [15] also used a rule based approach, where the thresholds used by his rules were learned using fuzzy logic Takahashi [16] has done some interesting work in developing individualized models of manual gear shifting for engine braking, while driving downhill.

Tribe [17] have developed warning systems which use active intervention, nudging the steering wheel in the proper direction to avoid a lane departure. There has also been work in predicting the effectiveness of warning systems, by Goodrich [18], Burgett [19] and Pape et al. [20] [21]. A great deal of attention is paid to individual performance and ability in the drowsy driver literature, by Knipling [22] and others. There have also been studies to determine where we look when we drive, by Land [23] [24].

\section{Methodology}

\subsection{Lane Detection}

The lane departure system is integrated in a prototype vehicle that can differ from the beneath it and give the appropriate result during its journey according to its position. To make use of the ability of the systems capability of differing colours beneath it the system can be implemented, that system can distinguish white line on a black ground which in real life situation represents the lane and its white marks. 


\subsection{System structure}

The vehicle is a four wheeled vehicle. The two back wheels each is attached to a twelve volts motor (Right motor and left motor), and two wheels are mounted in the front of the vehicles. The two rear motors are clearly responsible for the robots steering over the line.

\section{Technique: 1}

In this case, the system is provided with a transmitter. This has two modules, which are as follows:

1. Lane Control Station

2. Vehicle Unit

\subsubsection{Lane Control Station}

The lane control detection will collect the information from the sensors on the road ways. The driver going on their lane in correct manner means the sensor won't take any actions and suppose the driver lose his /her concentration means the vehicle will cross the definite lane and at that time the sensors will immediately sense and will give the required information to the control station as shown in figure 2.

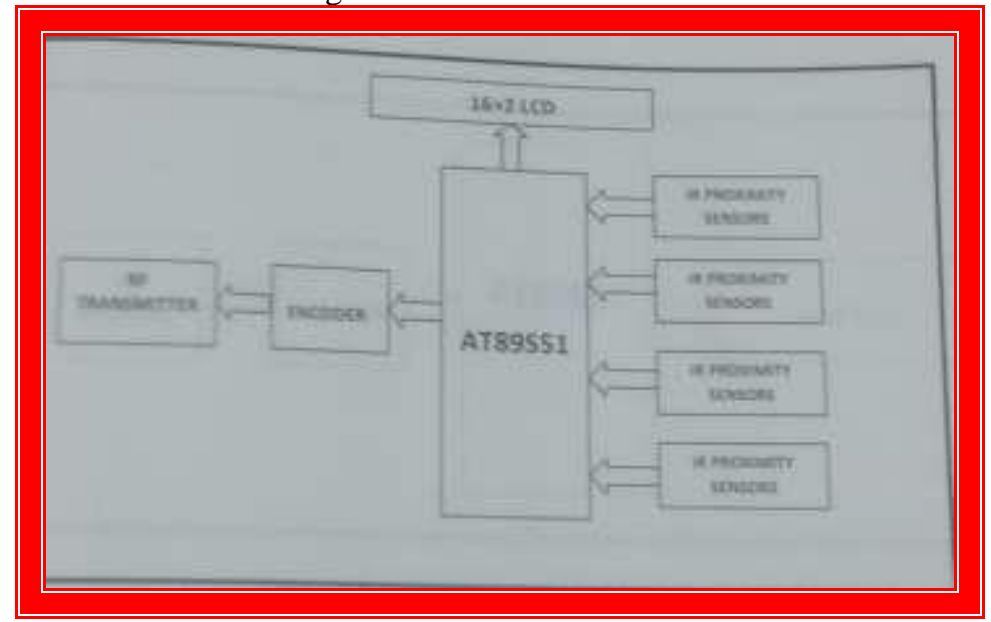

Figure 2: The Lane control station

\subsubsection{Vehicle Unit}

The driver side equipment is having a RF receiver which is fitted into the vehicle. The vehicle going through the wrong lane means the lane control station will send confirmation to the receiver, once the information is sent; the information will be displayed on the LCD in front of the driver as shown in figure 3.

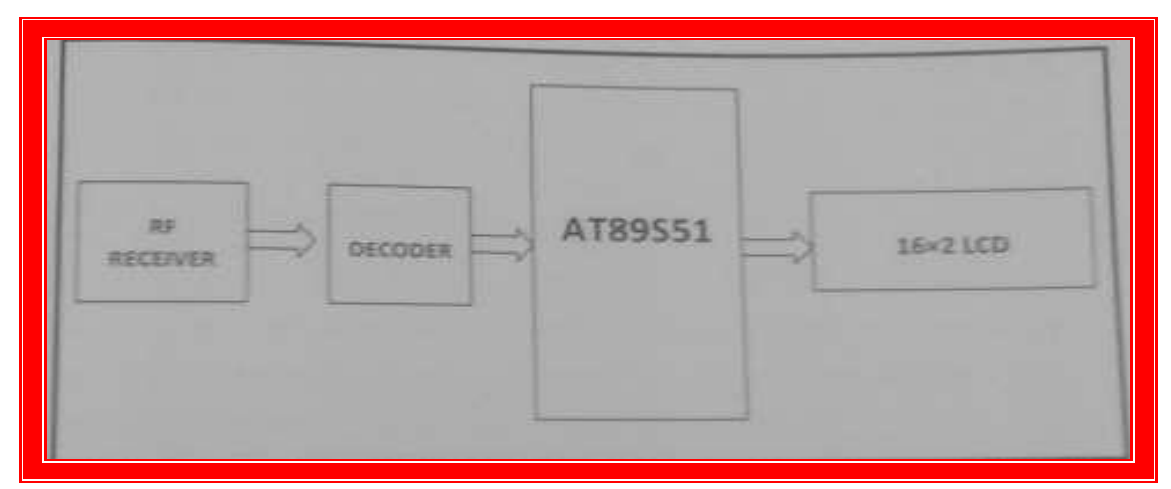

Figure 3:The vehicle unit

Technique: 2 In this case the system doesn't use the transmitter/receiver. Here in this case when the vehicle is running on roads where the lane control station is not installed then also the vehicle is able to detect the lanes with the help of IR sensors and will be able to intimate the driver about the lane change. The driver vehicle interface uses a visual indicator to indicate the lane departure.

\section{Sensor Analysis}

\subsection{Sensors in general}

It is important for each sensor to describe its performance. This can be classified into four groups:

a. Static characteristics - describing the performance with respect to very slow changes;

b. Dynamic characteristics - describing the performance with respect to variations in time and in the quantity that has to be measured;

c. Environmental characteristics - relating sensor performance after or during exposure to specified external conditions; 
d. Reliability characteristics - describing the sensor life expectancy; alongside these performance descriptions, specifications for sensor errors need to be defined:

- Sensitivity;

- Non-linearity and hysteresis;

- Resolution;

- Accuracy;

- Offset and zero drift;

- Noise;

- Response time;

- Frequency response;

To have such a detailed description about the sensors requires a lot of time, study and knowledge. Since a lot of detailed information is hidden from the public, the information provided in this report tries to be as complete as possible. A lot of systems are present and the amount of system models is almost unlimited. In this view the car is seen as the main system. In the system the driver is present. This system interacts with the environment. Three different functions of the system can be clearly distinguished:

a. Information has to be gathered;

b. Information needs to be evaluated;

c. A safety measure needs to be taken;

These functions can be referred to as Sense (1), Think (2), Act (3). In this report only the Sense sensors are reviewed and only the systems in which the driver is inside the loop. This means the driver has some overruling power over the systems

\subsection{Infrared Sensors or IR Sensors}

Infrared radiation is the portion of electromagnetic spectrum having wavelengths longer than visible light wavelengths, but smaller than microwaves, i.e., the region roughly from $0.75 \mu \mathrm{m}$ to $1000 \mu \mathrm{m}$ is the infrared region. Infrared waves are invisible to human eyes. The wavelength region of $0.75 \mu \mathrm{m}$ to $3 \mu \mathrm{m}$ is called near infrared, the region from $3 \mu \mathrm{m}$ to $6 \mu \mathrm{m}$ is called mid infrared and the region higher than $6 \mu \mathrm{m}$ is called far infrared. (The demarcations are not rigid; regions are defined differently by many).There are different types of IR sensors working in various regions of the IR spectrum but the physics behind "IR sensors" is governed by three laws: a. Planck's radiation law:

Every object at a temperature $\mathrm{T}$ not equal to $0 \mathrm{~K}$ emits radiation. Infrared radiant energy is determined by the temperature and surface condition of an object. Human eyes cannot detect differences in infrared energy because they are primarily sensitive to visible light energy from 400 to $700 \mathrm{~nm}$. Our eyes are not sensitive to the infrared energy. b. Stephan

\section{Boltzmann Law}

The total energy emitted at all wavelengths by a black body is related to the absolute temperature as

$$
\begin{aligned}
& W_{b}=\sigma T^{4} \quad \text { Where, } \begin{array}{l}
\text { Wb: total energy emitted } \\
\text { : constant }=5.67 \times 10^{-8} \mathrm{~m}^{-2} \mathrm{~K}^{-4} \\
\text { T: Temperature of the object }
\end{array}
\end{aligned}
$$

\section{c. Wein's Displacement Law}

Wein's Law says that objects of different temperature emit spectra that peak at different wavelengths. It provides the wavelength for maximum spectral radiant emittance for a given temperature.

\subsection{Elements of infrared Detection system}

A typical system for detecting infrared radiation is given in the following block diagram is shown in figure 4.

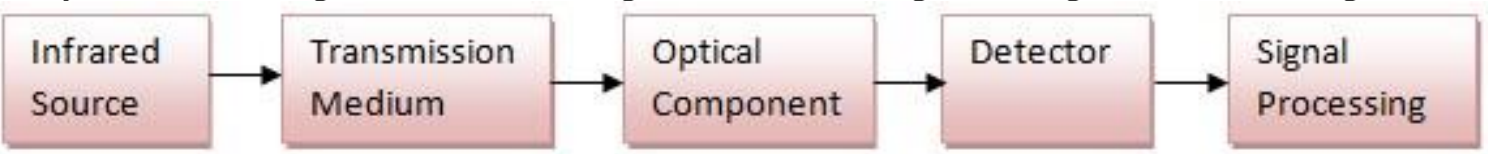

Figure 4: Block diagram for detecting infra-red radiation

\section{a. Infrared Source}

All objects above $0 \mathrm{~K}$ radiate infrared energy and hence are infrared sources. Infrared sources also include blackbody radiators, tungsten lamps, silicon carbide, and various others. For active IR sensors, infrared Lasers and LEDs of specific IR wavelengths are used as IR sources.

\section{b. Transmission Medium}

Three main types of transmission medium used for Infrared transmission are vacuum, the atmosphere, and optical fibers. The transmission of IR - radiation is affected by the presence of $\mathrm{CO}_{2}$, water vapour and other elements in the atmosphere. Due to absorption by molecules of water carbon dioxide, ozone, etc. the atmosphere highly attenuates most IR wavelengths leaving some important IR windows in the electromagnetic spectrum; these are primarily utilized by thermal imaging/ remote sensing applications. 
- Medium wave IR (MWIR: 3-5 $\mu \mathrm{m}) \cdot$ Long

wave IR (LWIR: 8-14 $\mu \mathrm{m})$

\section{c. Optical Components.}

Often optical components are required to converge or focus infrared radiations, to limit spectral response, etc. To converge/focus radiations, optical lenses made of quartz, $\mathrm{CaF} 2, \mathrm{Ge}$ and $\mathrm{Si}$, polyethylene Fresnel lenses, and mirrors made of $\mathrm{Al}$, Au or a similar material are used. For limiting spectral responses, band pass filters are used. Choppers are used to pass/ interrupt the IR beams.

\section{d. Infrared detectors.}

Various types of detectors are used in IR sensors. Important specifications of detectors are • Photosensitivity or Responsivity

Responsively is the Output Voltage/Current per watt of incident energy. Higher the better.

- Noise Equivalent Power (NEP)

NEP represents detection ability of a detector and is the amount of incident light equal to intrinsic noise level of a detector. - Detectivity (D*: D-star)

$\mathrm{D}^{*}$ is the photosensitivity per unit area of a detector. It is a measure of $\mathrm{S} / \mathrm{N}$ ratio of a detector. $\mathrm{D}^{*}$ is inversely proportional to NEP. Larger $D^{*}$ indicates better sensing element. In addition, wavelength region or temperature to be measured, response time, cooling mechanism, active area, no of elements, package, linearity, stability, temperature characteristics, etc. are important parameters which need attention while selecting IR detectors.

\section{e. Signal Processing}

Since detector outputs are typically very small, preamplifiers with associated circuitry are used to further process the received signals.IR Sensors work by using a specific light sensor to detect a select light wavelength in the Infra-Red (IR) spectrum. By using an LED which produces light at the same wavelength as what the sensor is looking for, you can look at the intensity of the received light. When an object is close to the sensor, the light from the LED bounces off the object and into the light sensor as shown in figure 5. This results in a large jump in the intensity, which we already know can be detected using a threshold.

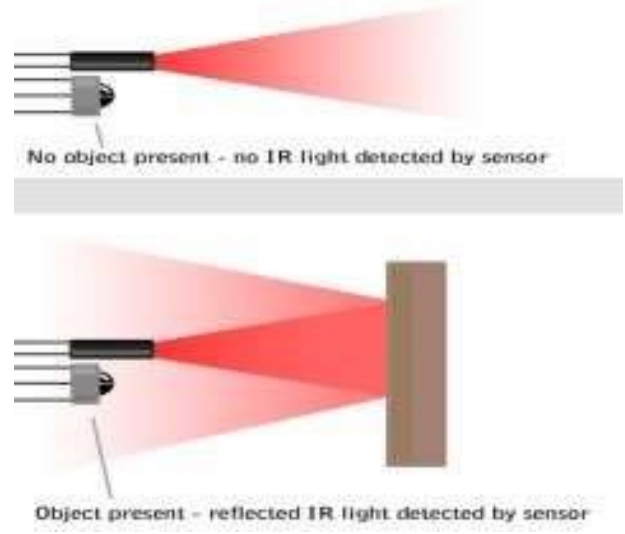

Figure 5: Depiction of the operation of an IR Sensor

\section{Technology and Materials}

\subsection{LDWS - lane departure warning system: working principle}

In the transmitter section, the microcontroller is interfaced with the LCD.The LCD is used to display the needed information (shown in figure 7). The sensor is interfaced in this transmitter section. Once the sensor is sensed the information is get by the liquid crystal display. After displayed the information is transmitted through the radio frequency comminucation. The encoder is used to encode the data before transmission. The LDWS is shown in figure 6.

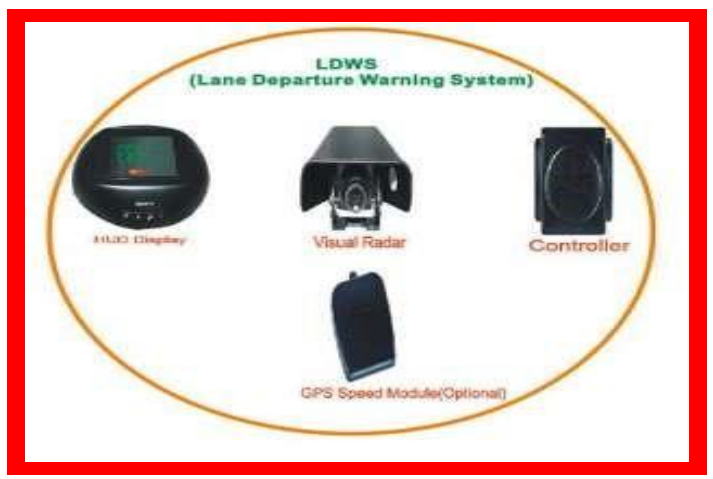

Figure 6: The Lane Departure Warning System 
In the receiver section, the Atmel microcontroller is used to receive the signal. The decoder is used to decode the data. The LCD is used to display the received data. The LED is used for visual identification. The lane control station will collect the information from the sensors on the road ways. If the vehicle is running smoothly in parallel ways to the lane, then there is no possibility of generation of any kind of alert or warning through the LDWS system. The system components are microcontroller, sensors, RF module, Encoder and Decoder and Chassis and other parts. The liquid-crystal display has the distinct advantage of having lower power consumption than the LED. It is typically of the order of microwatts for the display in comparison to the same order of mill watts for LEDs. The Low power consumption requirement has made it compatible with MOS integrated logic circuit. Its other advantages are its low cost, and good contrast. The main drawbacks of LCDs are additional requirement of light source, a limited temperature range of operation (between 0 and $60^{\circ} \mathrm{C}$ ), low reliability, short oper-ating life, poor visibility in low ambient lighting, slow speed and the need for an ac drive.

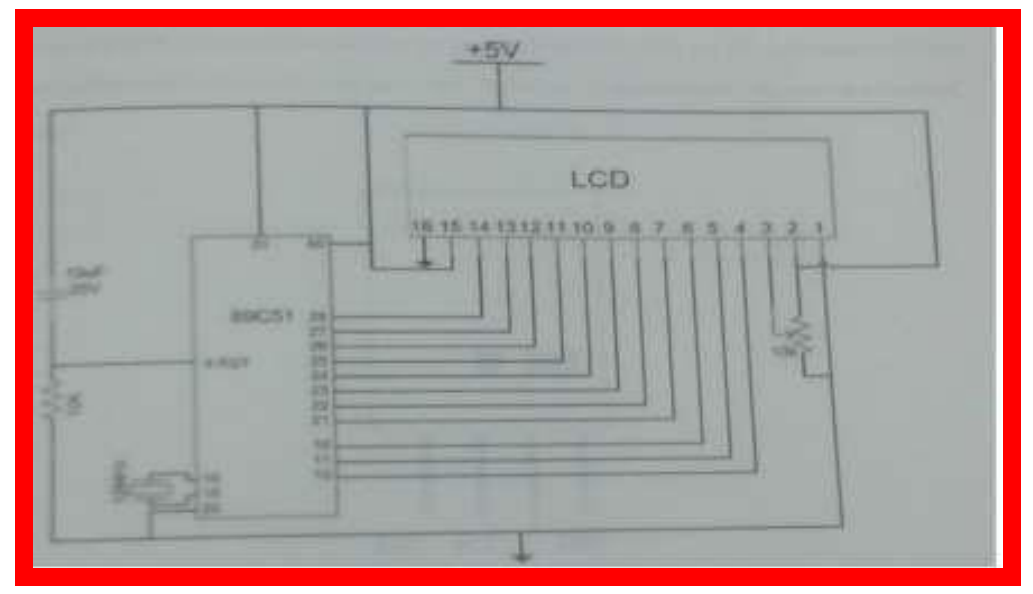

Figure 7: Circuit diagram of LCD

\subsection{Principle of work}

Through the camera mounted on windshield of a vehicle, acquiring and analyzing road traffic lines, when the vehicle deviates from the lane (unconscious: Deviate Road without turning signals), ahead of 0.5-1 second, the system alarms, assist the driver correct direction and initiatively prevent the occurrence of sideimpact, side-rollover and other accidents.

\subsubsection{Functions}

1. Accurately detecting road markings, when the vehicle has unconscious (did not turn cornering lamp) lane departure, it warns ahead of $0.5-1$ second.

2. When at the entrance and exit of the tunnel, before the eyes adjust their pupil light to be good, the system timely monitor road condition and front vehicles.

3. Maintain the vehicle running at a single lane and prevent occurrence of lane deviation due to monotony or fatigue driving at high-speed way, to avoid high-speed accident.

4. Advanced dynamic head-up display mode makes it available that the driver can observe important information on the traffic lane and front road condition and avoid inside and outside visual conflicts caused by alternate observation.

5. Timely detection of car battery voltage prevents occurrence of halfway breakdown due to lack of electricity or caused by battery failure.

6. Obtaining speed signal by GPS, simplifying installation, and the system does not destroy the original lines of vehicle; customers can make DIY installation.

7. The system alarms when vehicle exceed the speed, avoiding rear-end collision risk at high-speed and being photographed for over-speed.

\subsection{AT89C51 Microcontroller}

AT89C51 is an 8-bit microcontroller and belongs to Atmel's 8051 family. ATMEL 89C51 has 4KB of Flash programmable and erasable read only memory (PEROM) and 128 bytes of RAM. It can be erased and program to a maximum of 1000 times. In 40 pin AT89C51, there are four ports designated as $\mathrm{P}_{1}, \mathrm{P}_{2}, \mathrm{P}_{3}$ and $\mathrm{P}_{0}$. All these ports are 8-bit bi-directional ports, i.e., they can be used as both input and output ports. Except $\mathrm{P}_{0}$ which needs external pull-ups, rest of the ports have internal pull-ups. When $1 \mathrm{~s}$ are written to these port pins, they are pulled high by the internal pull-ups and can be used as inputs. These ports are also bit addressable and so their bits can also be accessed individually. Port $\mathrm{P}_{0}$ and $\mathrm{P}_{2}$ are also used to provide low byte and high byte addresses, respectively, when connected to an external memory. Port 3 has multiplexed pins for special functions like serial communication, hardware interrupts, timer inputs and read/write operation from external memory. AT89C51 has an inbuilt UART for serial communication. It can be programmed to operate at different baud rates. Including two timers \& hardware interrupts, it has a total of six interrupts. The Atmel AT89 series is an Intel 8051-compatible family of 8 bit microcontrollers $(\mu \mathrm{Cs})$ manufactured by the Atmel Corporation.

Based on the Intel 8051 core, the AT89 series remains very popular as general purpose microcontrollers, due to their industry standard instruction set, and low unit cost. This allows a great amount of legacy code to be reused without 
modification in new applications. While considerably less powerful than the newer AT90 series of AVR RISC microcontrollers, new product development has continued with the AT89 series for the aforementioned advantages. More recently, the AT89 series has been augmented with 8051-cored special function microcontrollers, specifically in the areas of USB, I ${ }^{2} \mathrm{C}$ (two wire interface), SPI and CAN bus controllers, MP3 decoders and hardware PWM. The Pin diagram of Atmel AT89S51is shown in figure

8 .

\subsubsection{Basic Pins}

a. PIN 9: PIN 9 is the reset pin which is used to reset the microcontroller's internal registers and ports upon starting up. (Pin should be held high for 2 machine cycles.)

b. PINS 18 \& 19: The 8051 has a built-in oscillator amplifier hence we need to only connect a crystal at these pins to provide clock pulses to the circuit.

c. PIN 40 and 20: Pins 40 and 20 are VCC and ground respectively. The 8051 chip needs $+5 \mathrm{~V} 500 \mathrm{~mA}$ to function properly, although there are lower powered versions like the Atmel 2051 which is a scaled down version of the 8051 which runs on $+3 \mathrm{~V}$.

d. PINS 29, $30 \& 31$ : As described in the features of the 8051, this chip contains a built-in flash memory. In order to program this we need to supply a voltage of $+12 \mathrm{~V}$ at pin 31 . If external memory is connected then PIN 31 , also called EA/VPP, should be connected to ground to indicate the presence of external memory. PIN 30 is called ALE (address latch enable), which is used when multiple memory chips are connected to the controller and only one of them needs to be selected. We will deal with this in depth in the later chapters. PIN 29 is called PSEN. This is "program store enable". In order to use the external memory it is required to provide the low voltage (0) on both PSEN and EA pins.

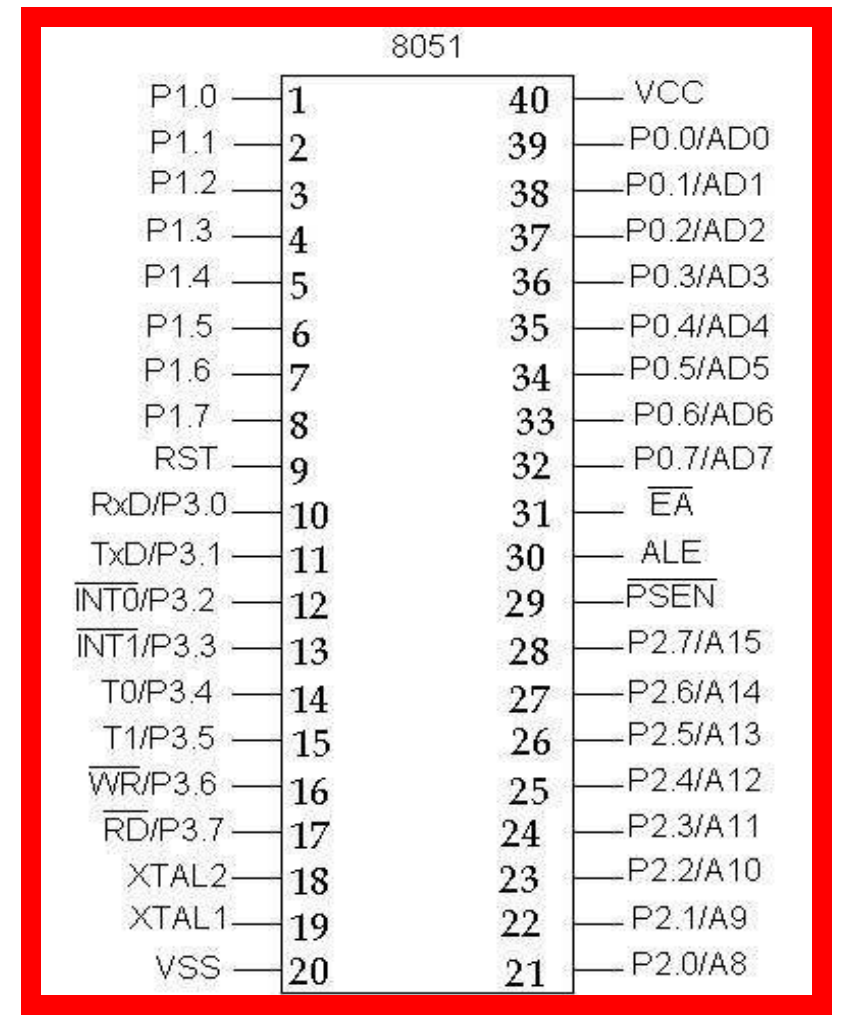

Figure 8: Pin diagram of Atmel AT89S51

\subsubsection{Ports}

There are 4 8-bit ports: P0, P1, P2 and P3.

a. PORT P1 (Pins 1 to 8): The port P1 is a general purpose input/output port which can be used for a variety of interfacing tasks. The other ports P0, P2 and P3 have dual roles or additional functions associated with them based upon the context of their usage. The port 1 output buffers can sink/source four TTL inputs. When 1 s are written to portn 1 pins are pulled high by the internal pull-ups and can be used as inputs.

b. PORT P3 (Pins 10 to 17): PORT P3 acts as a normal IO port, but Port P3 has additional functions such as, serial transmit and receive pins, 2 external interrupt pins, 2 external counter inputs, read and write pins for memory access.

c. PORT P2 (pins 21 to 28): PORT P2 can also be used as a general purpose 8 bit port when no external memory is present, but if external memory access is required then PORT P2 will act as an address bus in conjunction with PORT P0 to access external memory. PORT P2 acts as A8-A15, as can be seen from fig 8.

d. PORT P0 (pins 32 to 39) PORT P0 can be used as a general purpose 8 bit port when no external memory is present, but if external memory access is required then PORT P0 acts as a multiplexed address and data bus that can be used to access external memory in conjunction with PORT P2. P0 acts as AD0-AD7, as can be seen from fig 8. 
e. PORT P10: asynchronous communication input or Serial synchronous communication output.

\subsubsection{Block diagram of 8051}

Microcontroller manufacturers have been competing for a long time for attracting choosy customers and every couple of days a new chip with a higher operating frequency, more memory and upgraded A/D converters appeared on the market. However, most of them had the same or at least very similar architecture known in the world of microcontrollers as "8051 compatible". The whole story has its beginnings in the far $80 \mathrm{~s}$ when Intel launched the first series of microcontrollers called the MCS 051. Even though these microcontrollers had quite modest features in comparison to the new ones, they conquered the world very soon and became a standard for what nowadays is called the microcontroller. The main reason for their great success and popularity is a skilfully chosen configuration which satisfies different needs of a large number of users allowing at the same time constant expansions (refers to the new types of microcontrollers). Besides, the software has been developed in great extend in the meantime, and it simply was not profitable to change anything in the microcontroller's basic core. This is the reason for having a great number of various microcontrollers which basically are solely upgraded versions of the 8051 family. The block diagram of 8051 is shown in figure 9 .

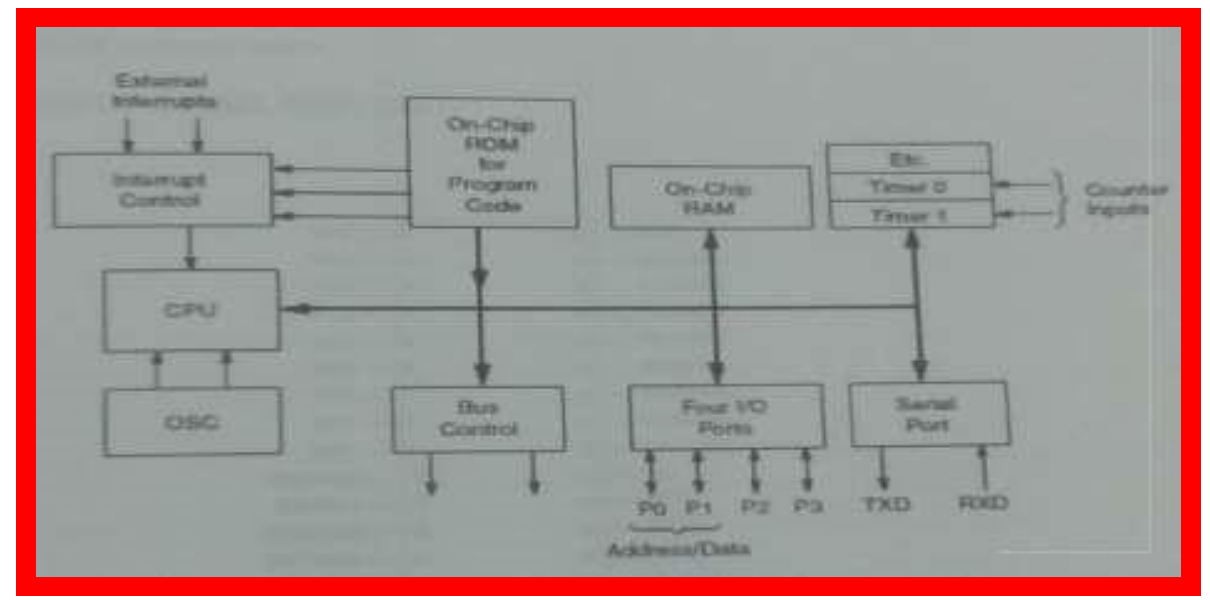

Figure 9: Block diagram of 8051

\section{Software}

The $\mathrm{C}$ programming language is a general-purpose programming language that provides code efficiency, elements of structural programming, and a rich set of operators is not a big language and is not designed for any one particular area of application. Embedded means some combination of computer hardware and programmable software which is specially designed for a particular task like displaying message on LCD. If you are still wondering about an embedded system, just take a look at these circuit applications using 8051 microcontroller. You can call these applications embedded systems as it involves hardware (8051 microcontroller) and software (the code written in assembly language).

1. Voltmeter using 8051

2. Thermometer using 8051

3. Frequency counters using AVR

Some real life examples of embedded systems may involve ticketing machines, vending machines, temperature controlling unit in air conditioners etc. Microcontrollers are nothing without a Program in it. One of the important parts in making an embedded system is loading the software/program we develop into the microcontroller. Usually it is called "burning software" into the controller. Before "burning a program" into a controller, we must do certain prerequisite operations with the program. This includes writing the program in assembly language or C language in a text editor like notepad, compiling the program in a compiler and finally generating the hex code from the compiled program. Earlier people used different software's/applications for all these 3 tasks. Writing was done in a text editor like notepad/WordPad, compiling was done using separate software (probably a dedicated compiler for a particular controller like 8051), converting the assembly code to hex code was done using another software etc. It takes lot of time and work to do all these separately, especially when the task involves lots of error debugging and reworking on the source code.

Keil Micro Vision is free software which solves many of the pain points for an embedded program developer. This software is an integrated development environment (IDE), which integrated a text editor to write programs, a compiler and it will convert your source code to hex files too.

Here is simple guide to start working with Keil $\mu$ Vision which can be used for

a. Writing programs in $\mathrm{C} / \mathrm{C}++$ or Assembly language

b. Compiling and Assembling Programs

c. Debugging program

d. Creating Hex and Axf file

e. Testing your program without Available real Hardware (Simulator Mode)

f. This is simple guide on Keil $\mu$ Vision 4 though also applicable on previous versions also.

g. These are the simple steps to get off the mark your inning! 
h. The Keil 8051 Development Tools are designed to solve the complex problems facing embedded software developers.

i. When starting a new project, simply select the microcontroller you use from the Device Database and the $\mu$ Vision IDE sets all compiler, assembler, linker, and memory options for you.

j. Numerous example programs are included to help you get started with the most popular embedded 8051 devices.

k. The Keil $\mu$ Vision Debugger accurately simulates on-chip peripherals ( ${ }^{2} \mathrm{C}, \mathrm{CAN}$, UART, SPI, Interrupts, I/O Ports, A/D Converter, D/A Converter, and PWM Modules) of your 8051 device. Simulation helps you understand hardware configurations and avoids time wasted on setup problems. Additionally, with simulation, you can write and test applications before target hardware is available.

1. When you are ready to begin testing your software application with target hardware, use the MON51, MON390, MONADI, or FlashMON51 Target Monitors, the ISD51 In-System Debugger, or the ULINK USB-JTAG Adapter to download and test program code on your target system

\section{Conclusion}

This system can be used as an intelligent driving system. The concept of vehicle sensor and lane sensor fusion is aimed at combining information from different sensors in order to get a more complete picture of the traffic environment and to ensure reliable operation under all circumstances. Those Sensors are capable of fulfilling these tasks can be categorized into passive sensors (e.g. optical sensors) and active sensors (e.g. radar, laser, sonar and near infra-red sensors).Passive sensors are well suited to lane detection as well as vehicle detection.However,their performance strongly depends on illumination conditions. Active sensors, on the other hand, are able to provide accurate information. Additionally; they function at any time of day and under practically any weather condition. It will allow the vehicle to stay in its lane during its whole course and it will intimate the driver if any lane shift occurs; this system will allow the driver correct its course if the vehicle left lane unintentionally. This system will also allow the vehicle to detect the lane in foggy condition or in rains etc. with the help of the RF module; it can also detect the lane using simple infra-red sensors.Automative technology matures, more and more functions of the underlying engine, gear box and even the steering wheel are no longer directly controlled by the driver, but rather via a computer, which receives instructions from the driver as inputs and delivers the desired effect.

\section{References}

[1] G. Jacobs, A. Aeron-Thomas, and A. Astrop, "Estimating global road fatalities,"Technical Report No. TRL 445, Australian National University, http://www.grsproad -safety.org/activities/reports/5/49.pdf, 2000.

[2] P. Batavia, "Driver-adaptive Lane departure warning systems," Ph.D. Dissertation, Robotics Institute, Carnegie Mellon University, Pittsburgh, PA, 1999.

[3] D. J. LeBlanc, G. E. Johnson, and P. J. Th. Venhovens, et al., "CAPC: an imple mentation of a road- departure warning system," in Proceedings of IEEE International Conference on Control Application, 1996, pp. 590-595.

[4] J. W. Lee, "A machine vision system for lane-departure detection," Computer Vision and Image Understanding, Vol. 86, 2002, pp. 52-78.

[5] H. Godthelp, P. Milgram, and G. J. Blaauw, "The development of a time-related measure to describe driver Strategy," Human Factors, Vol. 26, 1984, pp. 257-268.

[6] M. Bertozzi, A. Broggi, and M. Cellario, et al., "Artificial vision in road vehicles," in Proceedings of the IEEE, Vol. 90, 2002, pp. 1258-1271.

[7] L.A. Pipes. An operational analysis of traffic dynamics. Journal of Applied Physics,24:271-281, 1953.

[8] Walter W. Wierwille, Gilbert A. Gagne, and James R. Knight. An experimental study of human operator

[9] Models and closed-loop analysis methods for high-speed automobile driving. IEEE Transactions on Human Factors in Electronics, 8(3):187-201, 1967.

[10] George A. Bekey, Gerald O. Burnham, and Jinbom Seo. Control theoretic models of human drivers in car following. Human Factors, 19(4):399-413, 1977.

[11] Robert E. Chandler, Robert Herman, and Elliot W. Montroll. Traffic dynamics: Studies in car following. Operations Research, 6:165-184, 1958.

[12] Petros A. Ioannou. Autonomous intelligent cruise control. IEEE Transactions on Vehicular Technology, 42(4):657-672, November 1993.

[13] osef Schumann, Jan Loewenau, and Karl Naab. The active steering wheel as a continuous support for the driver's lateral control task. In Proceedings of Vision in Vehicles V, pages 1-8, 1995.

[14] R. Onken and J.P. Feraric. Adaptation to the driver as part of a driver monitoring and warning system. In The Second International Conference on Fatigue and Transportation: Engineering, Enforcement, and Education Solutions. February 1996.

[15] Kazunori Isomoto, Tadayuki Nibe, Takamasa Suetomi, and Tetsuro Butsuen. Development of a lane- keeping system for lane departure avoidance. In Proceedings of the 2nd World Congress on Intelligent Transportation Systems, number 2, pages 1266-1271, Yokohama, Japan, November 1995.

[16] Thomas E. Pilutti. Lateral Vehicle Co-Pilot To Avoid Unintended Roadway Departure. PhD thesis, The University Of Michigan, 1997.

[17] Hiroshi Takahashi and Kouichi Kuroda. A study on automated shifting and shift timing using and driver's mental model. In Proceedings, Intelligent Vehicles, 1996.

[18] R. Tribe, K. Prynne, and I. Westwood. Intelligent driver support. In Proceedings of the $2^{\text {nd }}$ World Congress On Intelligent Transportation Systems, pages 1187-1192, Yokohama, Japan, Nov 1995. 
[19] Michael A. Goodrich and Erwin R. Boer. Validating the satisficing approach to collision avoidance system design. In Proceedings of CESA '98, 1998.

[20] August Burgett. DOT's approach to its safety evaluation. In Proceedings of the Safety Evaluation of Intelligent Transportation Systems Workshop, pages 61-77, Reston, VA, May 1995.

[21] Doug Pape, Jeff Hadden, V.K. Narendran, Nathan Brown, Jeff Everson, Dean Pomerleau, and Charles

[22] Thorpe. Effectiveness analysis of run-off-road countermeasures for passenger vehicles on freeways and Two-lane secondary roads. Available from US Department of Transportation, National Highway Traffic Safety Administration, Office of Crash Avoidance Research, Washington, DC, 20590.

[23] Doug Pape, Jeff Hadden, V.K. Narendran, Nathan Brown, Jeff Everson, Dean Pomerleau, and Charles

[24] Thorpe. Effectiveness analysis of run-off-road countermeasures for heavy trucks. Available from US Department of Transportation, National Highway Traffic Safety Administration, Office of Crash Avoidance Research, Washington, DC, 20590.

[25] Ronalrd R. Knipling and Walter W. Wierwille. Vehicle-based drowsy driver detection: Current status and Future prospects. In Proceedings of IVHS America, pages 245-256, April 1994.

[26] M.F. Land and D.N. Lee. Where we look when we steer. Nature, 369:742-744, June 1994.

[27] Michael Land and Julia Horwood. Which parts of the road guide steering. Nature, 377:339-340, September 1980. 